\title{
Wirtschaftsprogramme gegen die Pandemiekrise - Deutschland im internationalen Vergleich
}

\author{
Die Wirtschaftsprogramme der 23 OECD-Länder setzen unterschiedliche Schwerpunkte. Im \\ Mittelpunkt der Programme stehen Geld- und Fiskalpolitik, Einkommensunterstützung und \\ Konjunkturprogramme, Arbeitsmarktpolitik und andere staatliche Programme in Bereichen \\ wie Infrastruktur, Gesundheit, Bildung und Klimaschutz. Im Vergleich scheint Deutschland \\ beim Schutz von Beschäftigung und Einkommen gut aufgestellt zu sein, wenn auch ohne \\ einen deutlichen Fokus auf die Armutsbekämpfung. Allerdings fehlt eine klare Vision zur \\ Ausgestaltung der Post-Corona-Ökonomie.
}

\begin{abstract}
Beim Vergleich der Wirtschaftspolitik zur Bekämpfung der Corona-Krise der OECD-Länder fällt auf, dass die wichtigsten staatlichen Konjunkturimpulse in den Politikfeldern Geld- und Fiskalpolitik sowie durch Hilfsmaßnahmen für Haushalte und Unternehmen, weitere Ausgabenprogramme und die Arbeitsmarktpolitik erfolgten. Seit dem Frühjahr 2020 haben die meisten Zentralbanken die Geldpolitik gelockert, um die Konjunktur anzukurbeln und die Kreditversorgung der Staaten und Unternehmen zu erleichtern. Ein weiterer, wohl auch nicht unerwünschter Nebeneffekt war die mögliche Abwertung der nationalen Währung, um die Exporte zu fördern und die Importkonkurrenz zu schwächen. Lockerung der Geldpolitik hieß in der Regel Senkung der Zinsen bzw. Beibehaltung schon vorher niedriger, manchmal sogar schon negativer Zinsen. Da die Pandemie in einer Phase bereits historisch niedriger Zinsen begann, war dieses Instrument nur begrenzt einsetzbar (Senkung um weniger als 2 Prozentpunkte). Japan und die Eurozone blieben bei den schon extrem niedrigen

(C) Der/die Autor:in(nen) 2021. Open Access: Dieser Artikel wird unter der Creative Commons Namensnennung 4.0 International Lizenz veröffentlicht (creativecommons.org/licenses/by/4.0/deed.de).

Open Access wird durch die ZBW - Leibniz-Informationszentrum Wirtschaft gefördert.

* Diese Studie wurde im Auftrag der Friedrich-Ebert-Stiftung erstellt.
\end{abstract}

Dr. Michael Dauderstädt ist freiberuflicher Publizist und Berater. Bis 2013 leitete er die Abteilung Wirtschafts- und Sozialpolitik der Friedrich-Ebert-Stiftung.
Zinsen (0 bzw. -0,1), wobei die Schweiz, Schweden und Dänemark de facto die EZB-Politik mit teils deutlich negativen Zinsen spiegeln. Deutschland als Teil der Eurozone genießt die gleichen geldpolitischen Impulse und profitiert mit seinen attraktiven Staatsschuldtiteln besonders vom Ankaufprogramm der EZB (vgl. Tabelle 1).

Angesichts der Grenzen der Zinspolitik setzten die Zentralbanken verstärkt auf das sogenannte Quantitative Easing, also den Kauf von Anleihen von Staaten und Unternehmen, um deren Liquidität, Solvenz und Kreditwürdigkeit zu stärken. Sichtbares Zeichen war die Ausdehnung der Zentralbankbilanzen, in deren Aktivseite die gekauften Vermögenstitel einflossen. Zwischen Frühjahr und Herbst 2020 nahmen viele Bilanzen um $20 \%$ bis $40 \%$ zu. Auch die Bilanz der Deutschen Bundesbank stieg um ca. $25 \%$ (von unter 2 Billionen Euro im April auf knapp 2,5 Billionen Euro im November 2020). Eine Ausnahme bildeten Dänemark, Norwegen und Island. Die Anleihenkäufe der EZB machen etwa $85 \%$ des gesamten erwarteten Haushaltsdefizits 2020/2021 der Eurozone von 1,7 Billionen Euro aus (IWF, 2020, 4).

Der unmittelbare Effekt war eine spektakuläre Erholung der Börsen, die im Sommer und Herbst 2020 wieder die Vorkrisenstände erreichten und oft überschritten. Diese Vermögenspreisinflation blieb isoliert. Eine Konsumpreissteigerung blieb weitgehend aus, aber eine deutliche Deflation konnte verhindert werden. Ein - von manchen Beobachter:innen befürchtetes - Wiederaufflammen der Inflation 2021 ist derzeit in den Markterwartungen nicht zu erkennen und wird daher von den Zentralbanken auch kaum als Risiko eingeplant. Keine Bank sieht für die nächste Zeit Zinserhöhungen vor. Die meisten Banken planen sogar noch weitere Ankäufe von Schuldtiteln. Da Staatsanleihen meist den Löwenanteil der Ankäufe ausmachen, trägt das zur weiteren Stabilisierung der Zinsbe- 
Tabelle 1

Zinspolitik ausgewählter Zentralbanken

Zinssätze in \%

\begin{tabular}{lrrrrrrr} 
Land & Q3 & Q4 & Q1 & Q2 & Q3 & Q4 & Q1 \\
& 2019 & 2019 & 2020 & 2020 & 2020 & 2020 & 2021 \\
\hline Australien & 1,00 & 0,75 & 0,25 & 0,25 & 0,25 & 0,25 & 0,10 \\
\hline Kanada & 1,75 & 1,75 & 0,25 & 0,25 & 0,25 & 0,25 & 0,25 \\
\hline Dänemark & $-0,75$ & $-0,75$ & $-0,60$ & $-0,60$ & $-0,60$ & $-0,60$ & $-0,60$ \\
\hline Eurozone & 0 & 0 & 0 & 0 & 0 & 0 & 0 \\
\hline Großbritannien & 0,75 & 0,75 & 0,10 & 0,10 & 0,10 & 0,10 & 0,10 \\
\hline Island & 3,50 & 3,00 & 1,75 & 1,00 & 1,00 & 1,00 & 0,75 \\
\hline Japan & $-0,10$ & $-0,10$ & $-0,10$ & $-0,10$ & $-0,10$ & $-0,10$ & $-0,10$ \\
\hline Korea & 1,50 & 1,25 & 0,75 & 0,50 & 0,50 & 0,50 & 0,50 \\
\hline Neuseeland & 1,00 & 1,00 & 0,25 & 0,25 & 0,25 & 0,25 & 0,25 \\
\hline Norwegen & 1,50 & 1,50 & 0,25 & 0 & 0 & 0 & 0 \\
\hline Polen & 1,50 & 1,50 & 1,00 & 0,10 & 0,10 & 0,10 & 0,10 \\
\hline Schweden & $-0,25$ & $-0,25$ & 0 & 0 & 0 & 0 & 0 \\
\hline Schweiz & $-0,75$ & $-0,75$ & $-0,75$ & $-0,75$ & $-0,75$ & $-0,75$ & $-0,75$ \\
\hline USA & 1,875 & 1,625 & 0,125 & 0,125 & 0,125 & 0,125 & 0,125
\end{tabular}

Quelle: BIS (o.D.)

lastung der Staatshaushalte bei und beugt einer Staatsschuldenpanik vor.

Fiskalpolitik: weiter expansiv, aber schwächer

Die durch die oben beschriebene Geldpolitik „entfesselte" Fiskalpolitik leistete den Hauptbeitrag bei der Krisenbekämpfung. Alle hier behandelten Länder haben einen - wenn auch unterschiedlich stark ausgeprägten - Wohlfahrtsstaat keynesianischer Prägung, bei dem in einer Krise zunächst die automatischen Stabilisatoren wirken, d.h. die Steuereinnahmen sinken und Ausgaben für Arbeitslosen- und Sozialhilfe steigen. In der Corona-Krise, die keine typische Rezession war, sondern sowohl angebots- wie nachfrageseitig wirkte, wurden diese automatischen Prozesse durch eine Fülle zusätzlicher Maßnahmen ergänzt und verstärkt.

Dazu zählen einnahmeseitig weitere Steuererleichterungen wie etwa auch Aufschübe bei der Zahlung von Steuern und Sozialabgaben und ausgabeseitig Zahlungen an Haushalte und Unternehmen sowie Bürgschaften und Kreditgarantien, wobei letztere den Staatshaushalt erst belasten, wenn tatsächlich der Kreditausfall eintritt. In vielen Ländern übertreffen diese Zusagen im Volumen die unmittelbaren Ausgaben, sodass der fiskalische Gesamtimpuls und die letztendliche Schuldenbelastung noch schwer abzuschätzen sind. Aber im ersten Ergebnis haben in allen Ländern gegenüber dem Vorjahr 2019 die Staatsausgaben beträchtlich zu- und die Einnahmen abgenommen.
Tabelle 2

Haushaltssalden und Schuldenstände

in Prozentpunkten des BIP

\begin{tabular}{|c|c|c|c|c|}
\hline \multirow[t]{2}{*}{ Land } & \multicolumn{2}{|c|}{$\begin{array}{l}\text { Veränderung } \\
\text { Haushaltssaldo }\end{array}$} & \multicolumn{2}{|c|}{$\begin{array}{l}\text { Veränderung } \\
\text { Schuldenstand }\end{array}$} \\
\hline & 2019-2020 & $2020-2021$ & 2019-2020 & 2020-2021 \\
\hline Australien & $-6,1$ & $-0,5$ & 12,1 & 10,4 \\
\hline Österreich & $-10,3$ & 3,2 & 13,4 & 3,2 \\
\hline Belgien & $-8,2$ & 2,9 & 16,2 & 1,6 \\
\hline Kanada & $-11,2$ & 2,9 & 9,6 & 4,0 \\
\hline Dänemark & $-7,2$ & 1,7 & 4,4 & 1,0 \\
\hline Finnland & $-3,8$ & 0,5 & 5,1 & 3,1 \\
\hline Frankreich & $-6,9$ & 2,7 & 15,0 & 1,7 \\
\hline Großbritannien & $-11,1$ & 1,7 & 18,5 & 3,4 \\
\hline Island & $-5,8$ & $-2,9$ & 8,3 & 6,0 \\
\hline Irland & $-5,8$ & $-0,2$ & 5,4 & 3,2 \\
\hline Italien & $-7,9$ & 0,7 & 19,9 & 2,2 \\
\hline Japan & $-9,5$ & 3,2 & 18,8 & 3,0 \\
\hline Korea & $-3,2$ & $-0,1$ & 6,4 & 4,5 \\
\hline Niederlande & $-8,1$ & 1,3 & 2,1 & 1,7 \\
\hline Neuseeland & $-3,4$ & 0,6 & 7,7 & 7,0 \\
\hline Norwegen & $-12,5$ & 6,8 & $-19,3$ & 11,2 \\
\hline Polen & $-7,5$ & 3,5 & 12,0 & $-0,3$ \\
\hline Portugal & $-6,2$ & 1,1 & 12,2 & 0,1 \\
\hline Spanien & $-8,6$ & 2,4 & 20,1 & 2,3 \\
\hline Schweden & $-4,5$ & 0,1 & 2,9 & 3,4 \\
\hline Schweiz & $-4,0$ & $-0,8$ & 3,2 & 1,9 \\
\hline USA & $-10,2$ & 0,8 & 20,2 & 5,8 \\
\hline Deutschland & $-5,7$ & $-1,3$ & 8,6 & 2,5 \\
\hline \multicolumn{5}{|c|}{ Durchschnitt, ohne } \\
\hline
\end{tabular}

Quelle: IWF (2020); eigene Berechnungen.

Damit stiegen die Haushaltsdefizite deutlich (vgl. 2. Spalte, Tabelle 2), was auch zu einem höheren Schuldenstand (außer in Norwegen) geführt hat (vgl. 4. Spalte, Tabelle 2). Diese Werte müssen aber mit Vorsicht beurteilt werden, da sie auch einen Rückgang des Bruttoinlandsprodukts (BIP) widerspiegeln, das ja bei den gewählten Indikatoren im Nenner steht. Für das Jahr 2021 planen die meisten Länder niedrigere Haushaltsdefizite (vgl. 3. Spalte, Tabelle 2), weil sie von einer Wiederbelebung der Konjunktur ausgehen, aber auch eine weitere Zunahme der Staatsverschuldung vermeiden oder zumindest bremsen wollen. Wie man beim Vergleich von Spalte 2 und 3 der Tabelle 2 sehen kann, würde aber in keinem Land das Defizit auf das Niveau von 2019 abgesenkt werden, sondern weiter deutlich darüber liegen. Das gilt besonders für Deutschland, dessen Defizit 2021 sogar über 2020 liegen dürfte. Im Durchschnitt nahm das Defizit von 2019 auf 2020 um über 7 Prozentpunkte des BIP 
Tabelle 3

\section{Hilfsprogramme}

\begin{tabular}{|c|c|c|c|}
\hline Land & Für Haushalte & Für Unternehmen & Sonstige Ausgabenprogramme \\
\hline Australien & Steuererleichterungen & Günstigere Abschreibungsregelungen; Verlustrücktrag & $\begin{array}{l}\text { Forschung und Entwicklung, Kli- } \\
\text { maschutz, Infrastrukturprojekte }\end{array}$ \\
\hline Österreich & $\begin{array}{l}\text { Einkommensteuersatzsenkung in der } 1 . \\
\text { Steuerklasse von } 25 \% \text { auf } 20 \%\end{array}$ & $\begin{array}{l}\text { Steuererleichterungen und Mehrwertsteuersenkung in ausge- } \\
\text { wählten Branchen und für bestimmte Investitionen; Notfallhilfen } \\
\text { für kleine Unternehmen; Umsatzverlustersatz (bis } 80 \% \text { ) }\end{array}$ & Klimaschutz, digitales Lernen \\
\hline Belgien & $\begin{array}{l}\text { Aussetzung der Bedienung von Hypothe- } \\
\text { kenkrediten }\end{array}$ & $\begin{array}{l}\text { Einkommensersatz für Selbständige; Kreditgarantien und Rück- } \\
\text { zahlungserleichterungen für Altkredite }\end{array}$ & \\
\hline Kanada & Steuerstundung (läuft aus) & Hilfen für Mieten, Kredite und Kreditgarantien & \\
\hline Dänemark & $\begin{array}{l}\text { Freigabe von Rentenzahlungen }(2,6 \% \\
\text { des BIP) }\end{array}$ & $\begin{array}{l}\text { Liquiditätshilfen, Kreditgarantien, gezielte Subventionen; Rekapi- } \\
\text { talisierungsfonds; Ersatz von Lohnfortzahlungen im Krankheitsfall }\end{array}$ & $\begin{array}{l}\text { Sozialer Wohnungsbau, kommu- } \\
\text { nale Ausgabenprogramme }\end{array}$ \\
\hline Finnland & & $\begin{array}{l}\text { Unterstützung kleine und mittlere Unternehmen und besonders } \\
\text { betroffene Branchen (Gastronomie, Reisen) }\end{array}$ & \\
\hline $\begin{array}{l}\text { Frank- } \\
\text { reich }\end{array}$ & $\begin{array}{l}\text { Hilfen für Obdachlose, Studierende; } \\
\text { Schulkinder }\end{array}$ & $\begin{array}{l}\text { Steuererleichterungen; Aussetzung von Steuer- und Sozialbeiträ- } \\
\text { gen; Stundung von Mieten und Versorgungskosten; Subventionen } \\
\text { für Autokauf; Hilfen für Automobil-, Tourismus- und Luftfahrtsek- } \\
\text { tor; Kreditgarantien und Quasi-Eigenkapitalbeteiligungen }\end{array}$ & $\begin{array}{l}\text { Öffentliche Investitionen (France } \\
\text { Relance Programm); Hilfspro- } \\
\text { gramm für Start-ups }\end{array}$ \\
\hline $\begin{array}{l}\text { Großbri- } \\
\text { tannien }\end{array}$ & $\begin{array}{l}\text { Einkommenshilfen Universal Credit und } \\
\text { Working Tax Credit erweitert, } 8 \text { Mrd. GBP }\end{array}$ & $\begin{array}{l}\text { Kreditprogramme: The Bounce Back Loan Scheme; Coronavirus } \\
\text { Business Interruption Loan Scheme, Covid Corporate Financing } \\
\text { Facility, Steuerstundung ( } 40 \text { Mrd. GBP) }\end{array}$ & $\begin{array}{l}\text { 31,9 Mrd. GBP für NHS; } 4 \text { Mrd. } \\
\text { GBP Bauinvestitionen, Zuschüs- } \\
\text { se an Bahn (3,5 Mrd. GBP) }\end{array}$ \\
\hline Island & $\begin{array}{l}\text { Freigabe von Zugriff auf Lebensversiche- } \\
\text { rungen }\end{array}$ & $\begin{array}{l}\text { Hilfen für Firmen mit mehr als } 75 \% \text { Umsatzeinbußen; Kreditga- } \\
\text { rantie für Icelandair }\end{array}$ & $\begin{array}{l}\text { Mehr öffentliche Investitionen, } \\
\text { vor allem digitale Infrastruktur }\end{array}$ \\
\hline Irland & $\begin{array}{l}\text { Einkommenshilfen für wegen der Pande- } \\
\text { mie Entlassene }\end{array}$ & $\begin{array}{l}\text { Steuerstundung, Kreditprogramme, sonstige Maßnahmen zur } \\
\text { Insolvenzvermeidung, Mehrwertsteuersenkung um } 2 \text { Prozent- } \\
\text { punkte; Steuererleichterungen für Selbständige }\end{array}$ & $\begin{array}{l}\text { Mehrausgaben für Gesundheit } \\
\text { und Brexit-Folgen }\end{array}$ \\
\hline Italien & $\begin{array}{l}\text { Kreditstundungen bei Erstwohnungskäu- } \\
\text { fen; Kindergeld; Steuerstundung }\end{array}$ & $\begin{array}{l}\text { Steuerstundung; Sozialbeiträge ausgesetzt, Garantien für alte } \\
\text { und neue Kredite }\end{array}$ & $\begin{array}{l}\text { Öffentliches Investitionspro- } \\
\text { gramm für grünes, digitales und } \\
\text { inklusives Wachstum }\end{array}$ \\
\hline Japan & $\begin{array}{l}\text { JPY } 100.000 \text { (= } 700 \text { Euro) Einmalzahlung } \\
\text { für jeden Einwohner; Extrazahlungen an } \\
\text { Alleinerziehende }\end{array}$ & $\begin{array}{l}\text { Direktzahlungen an besonders betroffene Geschäfte; Mietsub- } \\
\text { ventionen }\end{array}$ & Förderung des Inlandstourismus \\
\hline Korea & Konsumhilfen für ärmere Haushalte & $\begin{array}{l}\text { Stundung von Steuern und Sozialbeiträgen; Kredithilfen für kleine } \\
\text { und mittlere Unternehmen }\end{array}$ & $\begin{array}{l}\text { Korean New Deal (digital and } \\
\text { green) }\end{array}$ \\
\hline $\begin{array}{l}\text { Nieder- } \\
\text { lande }\end{array}$ & $\begin{array}{l}\text { Einkommenshilfen für Selbständige } \\
\text { (max. } 1.500 \text { Euro); Hilfen für Eltern, ohne } \\
\text { Kinderbetreuung }\end{array}$ & $\begin{array}{l}\text { Lohngarantien und Zuschüsse für Kleinunternehmen; Steuer- } \\
\text { stundung bis 2022; keine Zinsen auf Steuerschulden; zusätzliche } \\
\text { Kreditlinien }\end{array}$ & $\begin{array}{l}800 \text { Mio Euro extra für Gesund- } \\
\text { heitssektor }\end{array}$ \\
\hline $\begin{array}{l}\text { Neusee- } \\
\text { land }\end{array}$ & & $\begin{array}{l}\text { Lohnsubventionen; Krediterleichterungen; Pauschalhilfen (4.000 } \\
\text { Euro) für geschlossene Geschäfte; Steuerstundungen }\end{array}$ & $\begin{array}{l}\text { New Zealand Upgrade Pro- } \\
\text { gramme }\end{array}$ \\
\hline Norwegen & Verlängerung von Einkommenshilfen & $\begin{array}{l}\text { Fixkostenersatz; Rückstellungen für Kreditausfallgarantien; Hilfen } \\
\text { für Luftfahrtbranche }\end{array}$ & $\begin{array}{l}\text { Mehrausgaben für Forschung } \\
\text { und Entwicklung }\end{array}$ \\
\hline Polen & Höhere Sozialleistungen und Renten & Liquiditätshilfen & Überregionale Infrastruktur \\
\hline Portugal & $\begin{array}{l}\text { Lohnerhöhungen für Beschäftigte im } \\
\text { Gesundheits- und Niedriglohnsektor; hö- } \\
\text { heres Arbeitslosengeld; Hilfen für Eltern } \\
\text { ohne Kinderbetreuung; Mietstundung }\end{array}$ & $\begin{array}{l}\text { Verzögerte Zahlung von Sozialbeiträgen, Mehrwert- und Unter- } \\
\text { nehmenssteuer; Kreditlinien für kleine und mittlere Unternehmen, } \\
\text { Tourismus, Reisebüros und alle Unternehmen, die keine Entlas- } \\
\text { sungen vornehmen }\end{array}$ & Digitalisierung und Klimaschutz \\
\hline Spanien & $\begin{array}{l}\text { Hilfen für schwache Mieter } \\
\text { Hilfen für Selbständige }\end{array}$ & $\begin{array}{l}\text { Kreditgarantien ( } 40 \text { Mrd. Euro) für Investitionen; Fonds (10 Mrd. } \\
\text { Euro) für strategische Unternehmen; Hilfen für Automobil-Touris- } \\
\text { mus und Transportbranchen; Kreditstundungen; Aussetzung von } \\
\text { Insolvenz }\end{array}$ & $\begin{array}{l}\text { Fonds für Regionalregierungen } \\
\text { für Gesundheitskosten (2,9 Mrd. } \\
\text { Euro })+ \text { Sondermedizinfonds } \\
\text { (1,4 Mrd. Euro) }\end{array}$ \\
\hline $\begin{array}{l}\text { Schwe- } \\
\text { den }\end{array}$ & Einkommenssteuersenkung & Senkung der Arbeitgeberbeiträge & $\begin{array}{l}\text { Infrastruktur und „green eco- } \\
\text { nomy“ }\end{array}$ \\
\hline Schweiz & $\begin{array}{l}\text { Einkommensersatzleistungen für } \mathrm{Be}- \\
\text { schäftigte und Selbständige }\end{array}$ & $\begin{array}{l}\text { Kreditgarantien und Steuerstundungen für notleidende Unter- } \\
\text { nehmen }\end{array}$ & \\
\hline USA & $\begin{array}{l}\text { Einmalzahlungen } 2020 \text { (1.200 US- } \$+500 \\
\text { US-\$/Kind) an Familien; 2021: } 600 \text { US-\$; } \\
\text { Aufstockung Medicare und food stamps; } \\
\text { Aussetzung Bedienung Studentenkredite }\end{array}$ & $\begin{array}{l}\text { (Teils nicht rückzahlbare) Kredite an kleine und mittlere Unterneh- } \\
\text { men; Steuerstundungen; Kreditlinien für notleidende Unterneh- } \\
\text { men (500 Mrd. US-\$) }\end{array}$ & $\begin{array}{l}\text { Zuschüsse an Bundesstaaten } \\
\text { und Gemeinden; } 117 \text { Mrd. } \\
\text { US-\$ für Krankenhäuser und } \\
\text { Veteranen }\end{array}$ \\
\hline
\end{tabular}


Tabelle 3 (Fortsetzung)

Hilfsprogramme

\begin{tabular}{|c|c|c|c|}
\hline Land & Für Haushalte & Für Unternehmen & Sonstige Ausgabenprogramme \\
\hline Deutschland & $\begin{array}{l}\text { Mehrwertsteuersenkung bis Ende 2020, } \\
7,7 \text { Mrd. Euro Erleichterung Zugang zur } \\
\text { Sozialhilfe (ALGII), 5,2 Mrd. Euro zusätz- } \\
\text { liche Kranken- und Pflegeversicherung, } \\
\text { Kinderbonus } 300 \text { Euro/Kind, „Sozialga- } \\
\text { rantie 2021“ (Deckelung), EEG-Umlage }\end{array}$ & $\begin{array}{l}\text { Steuerstundungen, Verlustrücktrag und Abschrei- } \\
\text { bungsregeln ( } 7,3 \% \text { des BIP), Wirtschaftsstabilisie- } \\
\text { rungsfonds mit Liquiditätshilfen und Kreditgarantien } \\
\text { ( } 24 \% \text { des BIP), Umsatzverlustersatz, } 25 \text { Mrd. Euro } \\
\text { Neustart, } 50 \text { Mrd. Euro für KMU und Selbständige, } \\
\text { Beteiligung bei Lufthansa und TUI }\end{array}$ & $\begin{array}{l}2 \text { Mrd. Euro für Start-ups, } 6 \text { Mrd. Euro } \\
\text { E-Mobilität, 4,9 Mrd. Kommunen, 4,4 } \\
\text { Mrd. Euro Künstliche Intelligenz, 4 } \\
\text { Mrd. Gesundheitssystem, } 4 \text { Mrd. Euro } \\
\text { Öffentliche Investitionen, 3,6 Mrd. } \\
\text { Euro Wasserstoff-Strategie, 2,1 Mrd. } \\
\text { Digitalisierung Verwaltung und mehr }\end{array}$ \\
\hline
\end{tabular}

Quellen: OECD (2020); Bruegel (2020); ILO (2020); diverse nationale Quellen.

zu (Deutschland etwas unterdurchschnittlich). Für 2021 erwartet der Internationale Währungsfonds (IWF) im Durchschnitt ein um 1,4 Prozentpunkte niedrigeres Defizit, das aber immer noch 6 Prozentpunkte über dem Niveau von 2019 liegen würde. Insofern bleibt die Fiskalpolitik weiter expansiv. Auch die Verschuldung stieg gegenüber 2019 durchschnittlich um knapp 10 Prozentpunkte an, wobei Deutschland mit 8,6\% etwas darunter liegt. Auch 2021 wird die Verschuldung in allen Ländern voraussichtlich weiter ansteigen (vgl. 4. Spalte, Tabelle 2). Letztlich machen die Länder eine Abkehr von der stark expansiven Fiskalpolitik von der weiteren Entwicklung der Pandemie und der Konjunktur abhängig. Die derzeitigen Prognosen hoffen auf eine Erholung in der zweiten Jahreshälfte 2021, um dann die diversen Unterstützungsprogramme zurückfahren zu können.

\section{Hilfsprogramme}

Die staatlich verordneten Einschränkungen trafen und treffen viele Haushalte und Unternehmen hart. Um deren Einkommensverluste zu kompensieren und dadurch die Konsumnachfrage der Haushalte und das Überleben der betroffenen Unternehmen zu sichern, legten die Regierungen zahlreiche Hilfsprogramme auf (vgl. Tabelle 3). Sie umfassten direkte Zahlungen, Erleichterungen bei Steuern und Sozialabgaben, Kredite und Bürgschaften sowie gelegentlich auch Unternehmensbeteiligungen. Zu diesen Hilfsprogrammen zählen auch die arbeitsmarktpolitischen Maßnahmen (vor allem Kurzarbeitsregelungen und die konventionelle Arbeitslosenversicherung), auf die gesondert eingegangen wird.

In der Summe sind diese Programme hauptsächlich für die steigenden Haushaltsdefizite (vgl. Tabelle 2) verantwortlich, da sie höhere Ausgaben und niedrigere Einnahmen (wegen der Steuererleichterungen) verursachen. In ihrer Masse sind sie strukturkonservativ, da sie versuchen, bestehende Einkommensstrukturen zu erhalten. Zukunftsweisende Maßnahmen finden sich in Spalte 3 der Tabelle 3 und konzentrieren sich auf Klimaschutz,
Digitalisierung, E-Mobilität, Infrastruktur, Forschung und Entwicklung. Nicht berücksichtigt sind hier die geplanten Maßnahmen mit Mitteln aus dem im Juli 2020 beschlossenen EU-Programm Next Generation EU, da sie erst im Laufe von 2021 zum Einsatz kommen.

Eine Sonderstellung nehmen die Ausgaben ein, die direkt zur Bekämpfung der Pandemie dienen (z.B. für Krankenhäuser oder Impfstoffe). Zwar sind generell die Ausgaben für Gesundheit während der Pandemie nicht gestiegen (EIU, 2020), da andere Behandlungen unterlassen wurden, aber es kommen noch erhebliche Ausgaben für Impfungen auf die Länder zu. Die Kosten für den Impfstoff allein können bis zu 70 Euro/Impfling betragen. Sollten nur $60 \%$ der Bevölkerung geimpft (um Herdenimmunität zu erreichen) werden, so liegt der Aufwand bei ca. 40 Euro/Einwohner. Für die USA sind das etwa 13 Mrd. Euro, für Deutschland über $3 \mathrm{Mrd}$. Euro, für große europäische Länder über 2 Mrd. Euro, für kleinere immer noch 400 Mio. Euro. Dazu kommen noch die Kosten der Impfaktion selbst.

\section{Arbeitsmarktpolitik}

Ein starker Anstieg der Arbeitslosigkeit war unvermeidlich angesichts des staatlich verordneten Lockdowns und der Zurückhaltung der Bevölkerung, bestimmte, kontaktintensive Dienstleistungen in Anspruch zu nehmen. Die Reaktion der Regierungen lässt sich in zwei Varianten unterteilen: Beschäftigungsstabilisierung, vor allem durch Kurzarbeit, und Verbesserung der traditionellen Arbeitslosenunterstützung. Bei der Beschäftigungsstabilisierung befürchten manche Fachleute eine zu starke Strukturkonservierung, die eine Reallokation der Arbeit behindert. Das einzige Land ohne solche Programme sind die USA. Vor allem in der Phase des Abklingens der Krise treten dazu Maßnahmen der Beschäftigungsförderung (z. B. Lohnsubventionen bei Neueinstellungen, Ausbildung). Dazu zählen im weiteren Sinne auch viele der Programme, die die Kaufkraft stabilisieren und private und öffentliche Investitionen fördern und so dazu beitragen, neue Jobs zu schaffen. 
Tabelle 4

Maßnahmen der Arbeitsmarktpolitik

\begin{tabular}{|c|c|c|c|}
\hline Land & Beschäftigungsstabilisierung & Verbesserung der Arbeitslosenunterstützung & Beschäftigungsförderung \\
\hline Australien & Jobkeeper-Programm & & Jobmaker-Programm \\
\hline Österreich & Kurzarbeit bis März 2021 & & \\
\hline Belgien & Zeitweise Entlassung & & \\
\hline Kanada & Lohnsubvention (CEWS) bis Mitte 2021 & $\begin{array}{l}\text { Einkommenssicherung durch Canada } \\
\text { Emergency Response Benefit (CERB) }\end{array}$ & \\
\hline Dänemark & $\begin{array}{l}\text { Jobsicherung ( } 8 \% \text { aller Beschäftigten) nur noch } \\
\text { begrenzt; neues Kurzarbeitsprogramm }\end{array}$ & & \\
\hline Finnland & Zeitweise Entlassung & $\begin{array}{l}\text { Zugang zur Arbeitslosenversicherung } \\
\text { erleichtert und auf Selbständige ausgedehnt }\end{array}$ & \\
\hline Frankreich & Verstärktes Kurzarbeitsprogramm & & Finanzierung für Ausbildung \\
\hline $\begin{array}{l}\text { Großbritan- } \\
\text { nien }\end{array}$ & $\begin{array}{l}\text { Jobsicherungsprogramm zahlt } 80 \% \text { der Löhne und läuft } \\
\text { bis } 31.3 .21\end{array}$ & & \\
\hline Island & Kurzarbeitsprogramm & & \\
\hline Irland & Lohnsubventionen erweitert auf Saisonarbeit & & \\
\hline Italien & Kündigungsverbot & & Erleichterung von Zeitverträgen \\
\hline Japan & Finanzierung von Sonderurlaub & & \\
\hline Korea & Lohnsubventionen & & \\
\hline Niederlande & $\begin{array}{l}\text { Jobsicherungsprogramm bis Juli } 2021 \text {; danach } \\
\text { langsamer Ausstieg aus Lohnsubventionen }\end{array}$ & & \\
\hline Neuseeland & $\begin{array}{l}\text { Jobsicherung durch Lohnsubvention (14 Mrd. NZD = } \\
4,5 \% \text { BIP) }\end{array}$ & & \\
\hline Norwegen & $\begin{array}{l}\text { Partieller Ersatz der Lohnfortzahlung durch staatliche } \\
\text { Lohnsubvention }\end{array}$ & & \\
\hline Polen & Jobsicherungsprogramm wird zurückgefahren & Mindestlohn erhöht & \\
\hline Portugal & $\begin{array}{l}\text { Jobsicherungsprogramm verlängert für 2021; Kreditlinien } \\
\text { für alle Unternehmen, die keine Entlassungen vornehmen }\end{array}$ & Erhöhung von Löhnen und Arbeitslosengeld & \\
\hline Spanien & $\begin{array}{l}\text { Kurzarbeit verlängert bis } 31.1 .21 \text {; Sozialversicherungs- } \\
\text { beiträge ausgesetzt ( } 9,1 \text { Mrd. Euro) }\end{array}$ & Erhöhung von Löhnen und Arbeitslosengeld & \\
\hline Schweden & Kurzarbeitergeld & Erhöhung des Arbeitslosengelds & $\begin{array}{l}\text { Trainingsangebot für Rand- } \\
\text { gruppen des Arbeitsmarkts }\end{array}$ \\
\hline Schweiz & Kurzarbeit ausgedehnt & $\begin{array}{l}\text { Arbeitslosenversicherung wird durch Fonds } \\
\text { aufgestockt }(1,7 \% \text { BIP) }\end{array}$ & \\
\hline USA & & $\begin{array}{l}\text { Aufstockung der Arbeitslosenhilfe; 2021: } \\
300 \text { US-\$/Woche bis März }\end{array}$ & \\
\hline Deutschland & Kurzarbeitergeld (23,5 Mrd. Euro) & Erleichterung bei Hartz IV & $\begin{array}{l}500 \text { Mio. Euro Ausbildungs- } \\
\text { beihilfen }\end{array}$ \\
\hline
\end{tabular}

Quellen: OECD (2020); Bruegel (2020); ILO (2020); diverse nationale Quellen.

Tabelle 4 gibt einen Überblick über die Maßnahmen in den ausgewählten Ländern. Wie man sieht, lag der Schwerpunkt in der Beschäftigungssicherung durch partielle staatliche Übernahme der Lohnzahlungen (Ausnahme: USA). Viele dieser Programme wurden über ihren ursprünglichen Zeitrahmen über 2021 hinaus verlängert. Es bleibt abzuwarten, welche beschäftigungspolitischen Strategien langfristig erfolgreicher sind. In jedem Fall sind Maßnahmen sinnvoll, die die Qualifizierung und Umschulung von Arbeitskräften unterstützen, um ihre Integration in eine - möglicherweise stark veränderte - Post-Corona-
Ökonomie zu erleichtern. Je weniger stark die Pandemie die Strukturen verändert, desto nützlicher sind Politiken, die auf den Erhalt von bestehenden Arbeitsplätzen setzen.

\section{Deutschland im internationalen Vergleich}

Deutschland ging ins Coronajahr 2020 mit einer im internationalen Vergleich guten Wirtschaftslage: Die Beschäftigung war hoch und die Arbeitslosigkeit relativ niedrig (3\%) trotz schwachen Wachstums 2019. Der Staatshaushalt war mehr als ausgeglichen („schwarze Null“) und der 
Tabelle 5

Deutschland im internationalen Vergleich

\begin{tabular}{|c|c|c|}
\hline Politikfeld & Deutschland & Vergleichsländer \\
\hline Geldpolitik & EZB-Politik sorgt für Negativrendite neuer Staatschulden & $\begin{array}{l}\text { Ähnliche Politik mit weniger deutlichen Effekten in schon stark } \\
\text { verschuldeten Ländern }\end{array}$ \\
\hline Fiskalpolitik & $\begin{array}{l}\text { Hohes Defizit (auch noch 2021) und entsprechender Schulden- } \\
\text { anstieg }\end{array}$ & $\begin{array}{l}\text { Große Länder (G7 außer Frankreich) expansiver, sonst oft vorsichti- } \\
\text { ger; öfter mittelfristig steigende Schuldenstandsquoten }\end{array}$ \\
\hline Hilfsprogramme & Einkommensstabilisierung dominiert & $\begin{array}{l}\text { Einkommensstabilisierung dominiert auch in den meisten Ländern } \\
\text { (weniger stark in Korea, Neuseeland) }\end{array}$ \\
\hline für Haushalte & $\begin{array}{l}\text { Hilfen außer Kinderbonus nur indirekt (Mehrwertsteuer, Energie, } \\
\text { Deckelung Sozialbeiträge) regressiv mit geringer Konsumwirkung }\end{array}$ & $\begin{array}{l}\text { Direktzahlungen (z.B. USA, Japan), Erleichterung bei Steuern und } \\
\text { Sozialabgaben }\end{array}$ \\
\hline für Unternehmen & $\begin{array}{l}\text { Umfangreiche Hilfen in unterschiedlichen Formaten; werden } \\
\text { nicht voll ausgeschöpft; eher strukturkonservativ }\end{array}$ & $\begin{array}{l}\text { Ähnliche Hilfen in leicht unterschiedlichem Mix, Erleichterungen/ } \\
\text { Stundungen von Steuern und Abgaben, Zuschüssen, Krediten etc. }\end{array}$ \\
\hline $\begin{array}{l}\text { Sonstige } \\
\text { Programme }\end{array}$ & $\begin{array}{l}\text { Diverse Investitionsprogramme; Einstieg in Digitalisierung und } \\
\text { Klimaschutz, relativ schwach ausgelegt; Mittelabfluss langsam }\end{array}$ & $\begin{array}{l}\text { Ähnliche Schwerpunkte; interessant: Wohnungsbau in Dänemark; } \\
\text { Volumen variieren }\end{array}$ \\
\hline Arbeitsmarktpolitik & Beschäftigungssicherung dominiert & $\begin{array}{l}\text { Beschäftigungssicherung dominiert in den meisten Ländern (Aus- } \\
\text { nahme USA) }\end{array}$ \\
\hline $\begin{array}{l}\text { Beschäftigungs- } \\
\text { sicherung }\end{array}$ & Kurzarbeit (wie schon 2009) erfolgreich & Viele Länder übernahmen Kurzarbeit von Deutschland \\
\hline $\begin{array}{l}\text { Arbeitslosen- } \\
\text { unterstützung }\end{array}$ & Nur relativ geringe Erleichterungen & $\begin{array}{l}\text { Viele Länder haben Zahlungen an Arbeitslose verbessert (z. B. } \\
\text { USA, Schweden, Portugal) }\end{array}$ \\
\hline $\begin{array}{l}\text { Beschäftigungs- } \\
\text { förderung }\end{array}$ & Ausbildungsbeihilfen & Schwachpunkt in vielen Ländern \\
\hline
\end{tabular}

Quelle: Eigene Erhebungen.

Schuldenstand niedrig (unter $60 \%$ des BIP). Zu Beginn der Pandemie im Frühjahr 2020 reagierte das Land etwas zögerlich, aber dann doch entschlossen, sodass in der ersten Welle relativ wenig Opfer zu beklagen waren. Der Konjunktureinbruch war trotzdem massiv. Die zweite, tödlichere Welle ab Oktober 2020 bedroht die Erholung. Die wirtschaftspolitische Reaktion brach mit der heiligen Kuh „schwarze Null“ und lieferte einen für Deutschland ungewöhnlich starken, schuldenfinanzierten Konjunkturimpuls, der durch die Geldpolitik der EZB massiv erleichtert wurde. Der Mix zwischen Einkommenssicherung und Modernisierung war eher konservativ. Einen detaillierten Vergleich nach Politikfeldern liefert Tabelle 5.

Allgemein ist festzuhalten, dass sich die Konjunkturprogramme innerhalb der OECD-Länder stark ähneln. Zwar gibt es in jedem Land andere Akzente und spezielle Programme, aber der grundsätzliche Instrumentenkasten in den diversen Politikfeldern ist weitgehend derselbe, wenn auch die Einsatzintensität variiert. Deutschland liegt im Mittelfeld und damit unter seinen Möglichkeiten angesichts der guten Ausgangslage. Eine solide Bewertung der Programme würde eine Wirkungsanalyse erfordern, für die bisher kaum belastbare Daten vorliegen. Eine Kausalzuordnung von Ergebnissen (z.B. Wachstumsund Arbeitslosigkeitsraten) zu Politiken wäre zusätzlich problematisch. Man könnte immer einwenden, dass es ohne diese Maßnahmen schlimmer gewesen wäre. Im Ländervergleich müsste außerdem die unterschiedliche Wirtschaftsstruktur, politische Handlungsfähigkeit und Corona-Betroffenheit der Vergleichsländer berücksichtigt werden. Daher seien hier nur einige vorläufige Bemerkungen zum deutschen Konjunkturpaket gemacht:

- Pluspunkte: Pragmatische Bereitschaft zu höheren Ausgaben und Staatsschulden; großzügige Kurzarbeitsregelung (traditionelle deutsche Stärke); einige Zukunftsinvestitionsprogramme (wie Digitalisierung, EMobilität, Klimaschutz).

- Minuspunkte: Hilfen ohne gezielte direkte Förderung des Konsums (Mehrwertsteuersenkung fast nutzlos); einseitiger Fokus auf Einkommens- und Beschäftigungssicherung ohne deutliche Priorisierung der sozial Schwachen; keine klare, kohärente Zukunftsstrategie für eine Post-Corona-Wirtschaft mit entsprechenden Investitionsprioritäten.

\section{Ausblick und Exit-Strategie}

Die meisten Länder haben angesichts der zweiten Welle und der absehbar noch länger andauernden Pandemie ihre Stützungsprogramme 2021 verlängert. Erst für das zweite Halbjahr rechnen viele Regierungen, internationale Organisationen und Forschungsinstitute mit einer Kon- 
junkturerholung. Dann können viele Programme auslaufen, womit die Staatsausgaben auf ihr normales Niveau zurückkehren und die Einnahmen bei wieder „normaler“ Besteuerung zunehmen. Umstritten ist allerdings das Tempo der Normalisierung. Eine zu schnelle Konsolidierung der Staatshaushalte könnte die aufkommende Konjunktur abwürgen. Diese Gefahr droht in Deutschland ab 2022 (nach der Bundestagswahl). Ähnliches gilt für die Geldpolitik. Die meisten Zentralbanken planen nur ein langsames Zurückfahren der Anleihekäufe und ein noch späteres Anheben der Zinsen, solange eher Deflations- als Inflationsgefahren drohen und die hochverschuldeten Staaten bei höheren Zinsen in eine Schuldenkrise geraten könnten.

Unklar ist auch der Weg zu einem Schuldenabbau, soweit er überhaupt ein wünschenswertes Ziel darstellt. Selbst eine längere Phase ausgeglichener Haushalte würde nur bei ordentlichem nominalen BIP-Wachstum die Schuldenstandsquote (= Schulden/BIP) langsam sinken lassen. Optionen eines schnelleren Schuldenabbaus durch Haushaltsüberschüsse würden Ausgabenkürzungen und/oder Steuererhöhungen erfordern. Beides könnte das Wachstum einbrechen lassen und eine Deflation auslösen, womit - entgegen dem eigentlichen Ziel - die Schuldenstandsquote wieder steigen würde (wie z. B. in Griechenland durch die Sparpolitiken ab 2010). Dies könnte eventuell vermieden werden, wenn die Steuererhöhungen weder Konsum noch Investitionen beeinträchtigen würden, was vor allem bei einer Besteuerung hoher Einkommen und Vermögen wahrscheinlich wäre. Noch radikalere Konzepte sehen eine Vermögensabgabe (etwa nach dem Modell des deutschen Lastenausgleichs nach dem Zweiten Weltkrieg) vor.

Oft wurde gefordert, die massiven Staatsausgaben in der Pandemie dafür einzusetzen, ohnehin notwendige
Modernisierungsprozesse umzusetzen oder zumindest anzustoßen („Building back better“). Diese Priorität reibt sich mit den etablierten Interessen, bestehende Einkommensquellen zu erhalten. Obendrein konkurrieren auch innerhalb dieses weit gefassten Imperativs unterschiedliche Ziele: Klimaschutz, bessere Vorbereitung auf künftige Pandemien, Digitalisierung (vor allem im Bildungs- und Gesundheitssystem), E-Mobilität, Forschung und Entwicklung und Behebung von Defiziten in der Infrastruktur. Sie alle finden sich in wechselnder Zusammensetzung in den meisten nationalen Programmen, haben aber quantitativ oft ein vergleichsweise geringes Gewicht gegenüber der Einkommenssicherung. Das gilt auch für Deutschland. Indirekt trägt aber auch die Einkommenssicherung zur Modernisierung der Wirtschaft bei, wenn etwa Haushalte die Finanzmittel für neue Bedürfnisse (z. B. Digitalisierung) einsetzen, die sie erhalten und nicht für herkömmliche Zwecke (wie Reisen, Veranstaltungen, Gastronomie etc.) ausgeben wollen oder können.

\section{Literatur}

BIS (o. D.), https://www.bis.org/cbanks.htm (18. März 2020).

Bruegel (2020), The fiscal response to the economic fallout from the coronavirus, https://www.bruegel.org/publications/datasets/covid-national-dataset/ (18. März 2020).

EIU (2020) Covid-19:the impact on healthcare expenditure, https:// www.eiu.com/n/campaigns/covid-19-the-impact-on-healthcareexpenditure/\#mktoForm_anchor (20.4.2021)

ILO (International Labour Organization) (2020), https://www.lo.org/ emppolicy/Whatsnew/WCMS_754961/lang--en/index.htm (18. März 2020).

IWF (2020), World Economic Outlook, https://www.imf.org/en/Publications/WEO/weo-database/2020/October/download-entire-database (18. März 2020)

OECD (2020), Tackling coronavirus (COVID-19), Policy responses, http:// www.oecd.org/coronavirus/en/policy-responses (18. März 2020).

Title: Economic Programmes Against the Pandemic Crisis - Germany in International Comparison

Abstract: This paper compares the economic programmes of 23 OECD countries with that of Germany. It focuses on monetary and fiscal policies, income support and stimulus, labour market policies and other government programmes in areas such as infrastructure, health, education and climate protection. In comparison, Germany looks well placed in protecting employment and income, albeit without a clear anti-poverty focus. However, it lacks a clear vision of the kind of post-Corona economy it wants to achieve.

JEL Classification: O57, E58, E62 